\title{
Details zum Arbeitsvertrag für Medizinische Praxisassistentinnen
}

\section{Rahmenbedingungen für Lohnverhandlungen}

Aufgrund der seit 1991 kantonal von den Ärztegesellschaften ausgearbeiteten Lohnempfehlungen für Medizinische Praxisassistentinnen werden keine gesamtschweizerischen Lohnempfehlungen mehr publiziert. Bei individuellen Lohnverhandlungen sollen für Medizinische Praxisassistentinnen die nachstehend aufgeführten Rahmenbedingungen berücksichtigt werden, nämlich:

\section{Ausbildung}

- Eidg. Fähigkeitszeugnis Medizinische Praxisassistentin bzw. Diplom der Verbindung der Schweizer Ärzte (Vignette) inkl. Röntgenbewilligung.

\section{Arbeitsbedingungen: Berechnungsgrundlagen}

- 42-Stunden-Woche im Jahresdurchschnitt

- 4 Wochen Ferien (Medizinische Praxisassistentinnen unter 20 und ab 50 Jahren: 5 Wochen)

Nennenswerte Abweichungen von diesen Bedingungen können auf den Lohn umgerechnet werden.

\section{Regionale Gegebenheiten}

Die Löhne sollen den regionalen Gegebenheiten angepasst werden.

\section{13. Monatslohn}

Am Jahresende ist der Medizinischen Praxisassistentin ein 13. Monatslohn auszurichten. Umfasst das Arbeitsverhältnis nicht das ganze Kalenderjahr, so ist er anteilsmässig zu bezahlen.

\section{Dienstalterszulagen und Reallohnerhöhungen}

Die Höhe einer Dienstalterszulage soll auch für 2022 ein Thema des jährlichen Qualifikationsgespräches bilden. Insbesondere sollen bei der Festlegung des Lohnes auch jede zusätzliche Verantwortung (z.B. Ausbilden von Lernenden) oder Weiterbildung (z.B. Dosisintensives Röntgen etc.) der Angestellten berücksichtigt und der Lohn dementsprechend angepasst werden.

Eine Vorlage für ein Mitarbeitergespräch finden Sie online bei der FMH, Rubrik Medizinische Praxisassistentinnen:

https://www.fmh.ch/mpa/mpa-schweiz/arbeitgeber/ arbeitsbedingungen.html

\section{Teuerungsausgleich}

Die Teuerung soll ausgeglichen werden. Wo der Teuerungsausgleich vertraglich vereinbart ist, muss dieser auf jeden Fall gewährt werden. Im Falle einer Minusteuerung ist keine Teuerung auszugleichen (LIKP September 2021: $+0,9 \%$, Oktober 2021: $+1,2 \%$, Indexbasis Dez. 2010).

\section{Teilzeitarbeit im Monatslohn}

Bei Teilzeitarbeit beträgt der Bruttolohn (bei 42 Wochenstunden als Berechnungsgrundlage) 1/42 eines vollen Monatslohnes, multipliziert mit der Anzahl der vereinbarten Wochenarbeitsstunden.

\section{Stundenlohn}

Bei sehr geringer und gleichzeitig unregelmässiger Arbeitszeit empfiehlt sich die Ausrichtung eines Stundenlohnes. Als Stundenlohnansatz empfehlen wir 6\% eines Monatslohnes für ein Vollzeitpensum (13. Monatslohn ist anteilsmässig darin enthalten). Zusätzlich muss auf diesem Ansatz ein Ferienanteil von 8,33\% ausgerichtet werden, der den üblichen 4 Wochen Ferien entspricht und auf jeder Lohnabrechnung separat auszuweisen ist (bei 5 Wochen Ferien 10,64\%, bei 6 Wochen Ferien 13,04\% etc.). Diese Berechnung gilt auch bei der Auszahlung von Überstunden.

Um eine Benachteiligung gegenüber einer Anstellung im Monatslohn zu vermeiden, empfehlen wir, eine Feiertagsentschädigung $\mathrm{zu}$ vereinbaren. Nach dem Arbeitsgesetz können die Kantone bis zu 8 Feiertage den Sonntagen gleichstellen. Dazu kommt der 1. August. Maximal ist somit von 9 Feiertagen pro Jahr auszugehen. $\mathrm{Zu}$ berücksichtigen ist dabei, dass einige Feiertage immer am gleichen Datum stattfinden (z.B. 1. Mai) und mit einem Wochenende zusammenfallen können. $\mathrm{Zu}$ dem sind Feiertage von Kanton zu Kanton verschieden. Um eine jährliche Neuberechnung für jeden Kanton zu vermeiden, ist deshalb von einem Schnitt von 7,5 Feiertagen auszugehen, welche auf einen Werktag fallen. Der Feiertagszuschlag soll gleich wie der Ferienzuschlag berechnet werden, wobei die Praxis mit 260 Arbeitstagen pro Jahr rechnet: 7,5 Feiertage / (260 Arbeitstage 7,5 Feiertage) $=2,97 \%$ des Bruttolohnes pro Stunde.

\section{9. Überstunden (Ziffer 2 des Mustervertrags)}

Wenn immer möglich, sollen die Überstunden durch 
Freizeit oder allfällige Mehrferien (= Ferien, welche über dem gesetzlichen Minimum von 4 Wochen liegen) gleicher Dauer ausgeglichen werden. Ist dies nicht möglich, kann ausnahmsweise gewählt werden zwischen folgenden Varianten:

- Entschädigung samt einem Lohnzuschlag von 25\% für Vollzeit- und Teilzeitangestellte (Vorzugsvariante FMH/SVA nur für Vollzeitangestellte).

- Bei Teilzeitarbeit werden Überstunden, solange sie zusammen mit dem Teilzeitpensum die betriebsübliche Arbeitszeit für eine Vollzeitangestellte nicht überschreiten, lediglich nach dem normalen Stundenansatz vergütet (Vorzugsvariante SVA). ${ }^{1}$

- Arbeitgeber und Med. Praxisassistentin können durch schriftliche Vereinbarung eine andere Regelung wählen.

Als Stundenlohnansatz gelten 6\% eines Monatslohns für ein Vollzeitpensum zuzüglich Ferienanteil (vgl. Ziffer 8 Stundenlohn).

\section{Für alle vereinbarten Löhne gelten fol- gende Bestimmungen und Empfehlungen:}

\section{Abzüge vom Bruttolohn}

- AHV, IV, EO, ALV: 6,4\% (AHV, IV, EO = 5,3\%, ALV = 1,1\%)

- Nichtberufsunfallversicherung (bei einer wöchentlichen Arbeitszeit von mehr als 8 Stunden): Abzug gemäss konkretem Versicherungsvertrag.

- Berufliche Altersvorsorge (2. Säule BVG): Arbeitnehmeranteil (maximal und normalerweise 50\%) des altersabhängigen Beitrages gemäss Versicherungsausweis.

\section{Erläuterungen zum Mustervertrag}

\section{Arbeitszeit und Arbeitszeiterfassung (Ziffer 2 des Mustervertrages)}

Gemäss Art. 46 ArG und Art. 73 ArGV 1 besteht eine gesetzliche Aufzeichnungspflicht der Arbeitszeit durch den Arbeitgeber. Es muss für jede Arbeitnehmerin nachvollziehbar sein, wann sie gearbeitet, die Pausen bezogen und die Arbeit beendet hat. Aufgrund von diesen Angaben kann überprüft werden, ob die Arbeitsund Ruhezeitvorschriften des Arbeitsgesetzes eingehalten wurden. Der Arbeitgeber ist verpflichtet, diese Arbeitszeitaufzeichnungen während 5 Jahren aufzubewahren (Art. 73 Abs. 2 ArGV 1).

Arbeitszeiterfassung heisst nicht in jedem Fall Stempeluhr. Es gibt auch andere bewährte Möglichkeiten, die Arbeitszeiten zu erfassen (z.B. Excel-Tabelle). Wird die Arbeitszeit jedoch von der Arbeitnehmerin mit einer Excel-Tabelle selbst erfasst, empfehlen wir, dass die Zeiterfassung vom Arbeitgeber visiert wird.
Ein Excel-Tool zur Arbeitszeiterfassung finden Sie online beim SVA (Schweizerischer Verband Medizinischer Praxis-Fachpersonen) und beim ARAM (Association Romande des Assistantes Médicales).

Wir empfehlen für die Arbeitszeiterfassung, dass die Arbeitszeit mit dem Eintreffen am Arbeitsplatz und mit der Aufnahme der tatsächlichen Arbeitsvorbereitung beginnt (z.B. Computer starten, Wartezimmer lüften oder Zeitschriften bereitlegen, ggf. Post abholen etc.), und nicht erst mit der völligen Bereitschaft der Arbeitnehmerin am Arbeitsplatz für den Empfang des ersten Patienten/der ersten Patientin. Ebenso gehören auch Arbeiten am Abend, welche zum Praxisbetrieb gehören (z.B. Aufräumen, Arbeitsinstrumente reinigen etc.), zur Arbeitszeit. Der Arbeitsweg gehört nicht zur Arbeitszeit (vgl. die Ausnahme beim Pikettdienst Ziffer 6.6 des Mustervertrages). Es liegt im Ermessen des Arbeitgebers, mit Erlass von Weisungen die speditive Aufnahme der Arbeit sicherzustellen.

Es gibt verschiedene Arbeitszeitmodelle. Neben der im Mustervertrag empfohlenen wöchentlichen Arbeitszeit ist auch eine Jahresarbeitszeitregelung denkbar. Mit der Jahresarbeitszeit wird der Spielraum für die Gestaltung der Arbeitszeit ausgeweitet. Jedoch ist auch hier die maximale Grenze von 50 Stunden pro Woche zu berücksichtigen. Dies sollte jedoch nicht die Regel sein. Grössere Abweichungen vom üblichen Wochenstundenplan bedürfen zudem der Absprache beider Parteien. Idealerweise sollten die Zeitkonti der Arbeitnehmerinnen am Jahresende ausgeglichen sein. Zum Jahresende bestehende Überstundenguthaben sollten aber spätestens bis zur Mitte des Folgejahres durch Freizeitbezug ausgeglichen werden. Dasselbe gilt für Ferienguthaben.

\section{Freie Halbtage (Ziffer 6.2 des Mustervertrages)} Gemäss Art. 21 ArG ist der Arbeitnehmerin mindestens jede Woche ein freier Halbtag zu gewähren, wenn die wöchentliche Arbeitszeit auf mehr als fünf Tage verteilt wird. Dies gilt als Mindestanspruch.

\section{Stillende Mütter (Ziffer 6.7 des Mustervertrages)} Stillenden Müttern ist die erforderliche Zeit zum Stillen freizugeben, und der Arbeitgeber muss, wenn im Betrieb gestillt wird, einen geeigneten Ort zur Verfügung stellen (Art. 34 ArGV 3). Stillenden Müttern muss bei einer täglichen Arbeitszeit von bis zu 4 Stunden $30 \mathrm{Mi}$ nuten Stillzeit an die bezahlte Arbeitszeit angerechnet werden, bei einer täglichen Arbeitszeit von mehr als 4 Stunden 60 Minuten und bei einer täglichen Arbeitszeit von mehr als 7 Stunden 90 Minuten. Es handelt sich bei diesen Bestimmungen nur um Mindestzeiten, die an die bezahlte Arbeitszeit anzurechnen sind. Die Arbeitnehmerin verfügt unabhängig davon, ob sie im 
2 Beschwerliche und gefährliche Arbeiten sind namentlich: Bewegen schwerer Lasten von Hand; Bewegungen und Körperhaltungen, die zu vorzeitiger Ermüdung

führen; Arbeiten, die mit Einwirkungen wie Stössen, Erschütterungen oder Vibrationen verbunden sind; Hitze $\left(\max .28^{\circ} \mathrm{C}\right)$, Kälte $(\mathrm{min}$ $-5^{\circ} \mathrm{C}$ ) und Nässe; Arbeiten unter Einwirkung schädlicher Strahlen oder von Lärm $\geq 85 \mathrm{~dB}$; Arbeiten unter Einwirkung schädlicher Stoffe (z.B. Chemikalien) oder Mikroorganismen; Belastende Arbeitszeitsysteme (Art. 62 ArGV 1).
Betrieb stillt oder zum Stillen den Arbeitsplatz verlässt, über dieselbe bezahlte Stillzeit. Verlässt sie den Arbeitsplatz zum Stillen, ist für den Weg keine Verlängerung der bezahlten Stillzeit vorgesehen. Anderslautende Abmachungen zwischen dem Arbeitgeber und der betroffenen Arbeitnehmerin sind jedoch möglich.

\section{Gesundheitsschutz bei Mutterschaft (Ziffer 6.9 des Mustervertrages)}

Grundsätzlich hat ein Betrieb mit gefährlichen oder beschwerlichen Arbeiten ${ }^{2}$ eine Risikobeurteilung vorzunehmen. Darin muss insbesondere festgehalten sein, welche Gefahren für eine schwangere Frau und stillende Mutter bestehen, wie diese Risiken vermieden werden können und welche Arbeiten während der Schwangerschaft und Stillzeit verboten sind. Der Arbeitgeber hat die Pflicht, die betroffene Mitarbeiterin über die Ergebnisse der Risikobeurteilung zu informieren. Kann eine gefährliche gesundheitliche Belastung für Mutter und Kind nur durch das Ergreifen geeigneter Schutzmassnahmen ausgeschaltet werden, ist deren Wirksamkeit periodisch, mindestens vierteljährlich zu überprüfen.

Eine Vorlage einer Risikobeurteilung des Arbeitsplatzes finden Sie online bei der FMH, Rubrik Mutterschutz in der Arztpraxis,

https://www.fmh.ch/mpa/mpa-schweiz/arbeitgeber/ mutterschutz.html

\section{Lohnfortzahlung bei Schwangerschaft und Niederkunft (Ziffer 8.1ff. des Mustervertrages)}

Es sind zwei Phasen zu unterscheiden: Die Schwangerschaft, bei der sich die Lohnfortzahlung nach Art. 324a des Schweizerischen Obligationenrechts (OR) richtet, und die Zeit nach der Niederkunft, bei der sich die Ansprüche nach dem Erwerbsersatzgesetz (EOG) richten. Vergleiche die Ergänzung zur gesetzlichen Lösung in Ziffer 8.3 Abs. 2 des Mustervertrages.

Der Anspruch auf Mutterschaftsentschädigung beginnt am Tag der Niederkunft und endet spätestens nach 14 Wochen bzw. 98 Tagen. Bei längerem Spitalaufenthalt des Kindes kann die Mutter beantragen, dass der Anspruch auf Entschädigung erst mit der Heimkehr des Kindes beginnt. Die Mutterschaftsentschädi-

FMH-Delegierte für MPA-Fragen: Dr. med. Aisha Ahmed, Präsidentin

- Association Genevoise des Assistantes et des Assistants Médicaux AGAM: Marie Estime Lorréus Gachet, Präsidentin

Associazione Ticinese Assistenti di studio Medico ATAM: Mary Carrera, Sekretariat

- Association Romande des Assistantes Médicales ARAM: Marie-Paule Fauchère, Präsidentin

- Schweizerischer Verband Medizinischer Praxis-Fachpersonen SVA: Dr. iur. Peter Burkhalter, Zentralsekretär

gung wird als Taggeld über die AHV-Ausgleichskasse ausgerichtet und beträgt $80 \%$ des vor der Niederkunft erzielten durchschnittlichen Erwerbseinkommens, höchstens aber 196 Franken im Tag.

Hat der Arbeitgeber eine Krankentaggeldversicherung abgeschlossen, kann die Arbeitnehmerin bei Krankheit bei der Versicherung die Differenz zwischen den erwähnten 196 Franken und dem Entschädigungsanspruch der Versicherung (80\% des versicherten Lohns) geltend machen. Und zwar für die Zeit der Erkrankung, aber maximal bis zum Ablauf des Mutterschaftsurlaubs. Sollte die Arbeitnehmerin auch nach Ablauf des Mutterschaftsurlaubs noch krank sein, hat sie Anspruch auf das gesamte Taggeld vom Taggeldversicherer.

Solange eine Mutterschaftsentschädigung ausbezahlt wird, kann kein Taggeld der Arbeitslosenversicherung, der IV oder der obligatorischen Unfallversicherung bezogen werden. Krankentaggelder der Krankentaggeldversicherung und der obligatorischen Unfallversicherung sind nicht AHV-beitragspflichtig, hingegen sind Erwerbsersatzleistungen gemäss EOG AHV-beitragspflichtig.

\section{Arbeitsverträge und weitere Anstellungs- bedingungen}

Wir empfehlen nachdrücklich die schriftliche Vertragsform auf dem von der FMH und den Berufsverbänden der Medizinischen Praxisassistentinnen gemeinsam erarbeiteten Vertragsformular mit zugehörigen kantonalen Empfehlungen; Bezugsquellen:

- Verbindung der Schweizer Ärztinnen und Ärzte FMH, Nussbaumstrasse 29, Postfach 300, 3000 Bern 15, Tel. 031359 11 11,www.fmh.ch, E-Mail:mpa@fmh.ch - Association Romande des Assistantes Médicales ARAM, 1003 Lausanne, Tel. 07938012 44/079 47168 53, www.aram-vd.ch, E-Mail: info@aram-vd.ch

- Schweiz. Verband Medizinischer Praxis-Fachpersonen SVA, Elfenstrasse 19, Postfach 1010, 3000 Bern 6, Tel. 03135690 09,www.sva.ch, E-Mail: sekretariat@ sva.ch

Die Berufsverbände der Medizinischen Praxisassistentinnen in Genf und im Tessin haben eine eigene Arbeitsvertragskonvention resp. Arbeitsvertrag; Bezugsquellen:

Associazione Ticinese Assistenti di studio Medico ATAM c/o M. Carrera, Via dei Faggi 2A, 6912 Lugano-Pazzallo, www.atam.ch, E-Mail: atam@bluewin.ch

Association Genevoise des Assistantes et des Assistants Médicaux AGAM, 1200 Genève, www.agam-ge.ch, E-Mail: presidente@agam-ge.ch

Die Löhne für Lernende richten sich nach den kantonalen Empfehlungen. 